\title{
Promoter prediction in the rhizobia
}

Correspondence

Turlough M. Finan

finan@mcmaster.ca

Received 4 December 2005

Revised 26 February 2006

Accepted 28 February 2006

\author{
Shawn R. MacLellan, Allyson M. MacLean and Turlough M. Finan \\ Center for Environmental Genomics, Department of Biology, McMaster University, 1280 Main \\ St West, Hamilton, Ontario L8S 4K1, Canada
}

\begin{abstract}
The ability to recognize and predict non- $\sigma^{54}$ promoters in the alphaproteobacteria is not well developed. In this study, 25 experimentally verified Sinorhizobium meliloti promoter sequences were compiled and used to predict the location of other related promoters in the S. meliloti genome. Fourteen candidate predictions were targeted for verification and of these at least 12 proved to be genuine promoters. As a result, the experimental identification of 12 novel promoters linked to genes $r p o D, \operatorname{top} A, r p m J$, $\operatorname{trp} S, \operatorname{rop} B 1, \operatorname{met} C, r p s T, \sec E, \operatorname{trkH}$ and three tRNA genes is reported. In all, 99 predicted and verified promoters are reported, including those linked with 13 tRNA genes, eight ribosomal protein genes and a number of other physiologically important or essential genes. On the basis of sequence conservation and a mutational analysis of promoter activity, the -35 and -10 consensus for these promoters is $5-C T T G A C-N_{17}-C T A T A T$. This promoter structure, which seems to be widely conserved amongst several other genera in the alphaproteobacteria, shares significant similarity with, but is skewed by a $1 \mathrm{nt}$ step from, the canonical Escherichia coli $\sigma^{70}$ promoter. Perhaps this difference is responsible for the observation that S. meliloti promoters are often poorly expressed in E. coli. In this regard, expression data from plasmid-borne gfp-reporter fusions to eight of the $S$. meliloti promoters verified in this work revealed that while these promoters were very active in S. meliloti and Agrobacterium tumefaciens only very low, near-background activity was detected in $E$. coli.
\end{abstract}

\section{INTRODUCTION}

Sinorhizobium meliloti is one of a large group of alphaproteobacteria that infect plant or animal cells and mediate symbiotic or pathogenic interactions within the host. In the case of S. meliloti, genes required for host invasion and nitrogen fixation are carried on a primary chromosome and two large (1.4 and $1.7 \mathrm{Mb}$ ) plasmids (Galibert et al., 2001). For several members of this group, including Agrobacterium tumefaciens, Brucella melitensis, B. suis, B. abortus, S. meliloti and Bradyrhizobium japonicum, genome sequences have been published (DelVecchio et al., 2002; Galibert et al., 2001; Halling et al., 2005; Kaneko et al., 2002; Paulsen et al., 2002; Wood et al., 2001). As is increasingly evident in organisms such as Escherichia coli, the initial annotation of the genome sequence misses many genes and some of these (especially small RNA genes) have been found by scanning the genome for promoter-like elements linked to transcriptional termination elements (Argaman et al., 2001; Chen et al., 2002). Promoter prediction in E. coli is particularly well established, where the canonical consensus for $\sigma^{70}$ promoters is based on sequence conservation amongst several hundred promoter sequences (Harley \& Reynolds, 1987; Lisser \& Margalit, 1993). Except for the $\sigma^{54}$ promoters (Dombrecht et al., 2002; Thony \& Hennecke, 1989), the ability to predict and recognize promoter elements is poorly developed in most members of the alphaproteobacteria. We have therefore compiled a collection of experimentally verified $S$. meliloti promoter sequences (Bae et al., 1989; Fisher et al., 1987; Gustafson et al., 2002; Leong et al., 1985; MacLellan et al., 2005; Osteras et al., 1995; Papp, 2004; Ronson et al., 1987) and have used these to identify a large number of related sequences in the genome. We predict that these sequences represent novel RpoD-dependent promoter elements and have confirmed that 12 of them represent genuine active promoters. An analysis of experimentally verified $S$. meliloti promoter sequences indicates that (1) the -10 region is particularly diverse in sequence and poorly defined except for two highly conserved residues, and (2) the consensus -10 and -35 hexanucleotides share a significant degree of conservation with the E. coli RpoD-dependent promoter consensus but these sequences are positionally skewed relative to one another by a single nucleotide step.

\section{METHODS}

Bacterial strains, plasmids and oligonucleotides. The bacterial strains and plasmids used in this study are listed in Table 1. Both $S$. meliloti and A. tumefaciens were grown in LB broth supplemented with $100 \mu \mathrm{g}$ streptomycin $\mathrm{ml}^{-1}$ and $30 \mu \mathrm{g}$ gentamicin $\mathrm{ml}^{-1}$ (where appropriate) at $30^{\circ} \mathrm{C}$ while $E$. coli was grown in the same medium supplemented with $5 \mu \mathrm{g}$ gentamicin $\mathrm{ml}^{-1}$ at $37^{\circ} \mathrm{C}$. Antibiotic concentrations were doubled in solid medium. The oligonucleotides used for primer extension reactions are also listed in Table 1.

Sequence analysis. The first-generation promoter prediction was based upon an alignment of previously verified $S$. meliloti promoter 
Table 1. Bacterial strains, plasmids and primer extension oligonucleotides used in this study

\begin{tabular}{|c|c|c|}
\hline Strain or plasmid & Characteristic or genotype & Source or reference \\
\hline E. coli $\mathrm{DH} 5 \alpha$ & supE44 $\Delta$ lacU169( $\phi 80$ lacZAM15) hsdR17 recA1 endA1 gyrA96 thi-1 relA1 & Strain collection \\
\hline A. tumefaciens At9023 & pATC58 deletion derivative of strain C58 ( Rif $^{\mathrm{r}} \mathrm{Sm}^{\mathrm{r}}$ ) & Strain collection \\
\hline \multicolumn{3}{|l|}{ Plasmids } \\
\hline pOT1 & $g f p U V$ transcriptional reporter plasmid $\left(\mathrm{Gm}^{\mathrm{r}}\right)$ & Allaway et al. (2001) \\
\hline pTH1985 & Site-directed mutant $(\mathrm{A} \rightarrow \mathrm{T}$ in -35 promoter region $)$ of $\mathrm{pTH} 1560$ & This work \\
\hline pTH1991 & Site-directed mutant $(\mathrm{C} \rightarrow \mathrm{T}$ in -35 promoter region $)$ of $\mathrm{pTH} 1560$ & This work \\
\hline pTH1982 & 189 bp PCR product encompassing incA 2 promoter cloned into pOT1 & This work \\
\hline pTH1983 & Site-directed mutant $(\mathrm{T} \rightarrow \mathrm{A}$ in -35 promoter region $)$ of $\mathrm{pTH} 1982$ & This work \\
\hline pTH1984 & Site-directed double mutant $(\mathrm{C} \rightarrow \mathrm{T}, \mathrm{T} \rightarrow \mathrm{A}$ in -35 promoter region $)$ of $\mathrm{pTH} 1982$ & This work \\
\hline rpmE & GATCTAGAGCAAGGCATTGCGACTTTGG & \\
\hline rpmJ & GATCTAGATCCTGATCCGCAGGTTTCG & \\
\hline $\operatorname{ptr} B$ & AGTCTAGACACGCAGCAGATTAAAGCG & \\
\hline metC & TATCTAGACTTCCCGATCTTGTTGTCTGC & \\
\hline $\operatorname{trpS}$ & AGTCTAGATACGAGCGGCTTGAATTCG & \\
\hline $\operatorname{trkH}$ & AGTCTAGATAGTGCCGTGCGTGACC & \\
\hline SMc02206 tRNA & AATCTAGAACCGACGACATCAAGCTTGG & \\
\hline SMc01378 tRNA & AGTCTAGACTGTCGGGATCAATCGACC & \\
\hline SMc00758 tRNA & AGTCTAGACTAACCTACTGAGCTACTCC & \\
\hline $\operatorname{rps} T$ & AGTCTAGACTGCGACCTGTCATACAAGC & \\
\hline ropB1 & AGTCTAGAGTACGCATACTATTCTCCTTTCG & \\
\hline
\end{tabular}

sequences (Fig. 1, sequences 1-11). These sequences or sequence 10 (the $r R N A$ operon promoter) were used to derive a search string that could be used to scan the S. meliloti genome for matches. A standard weight matrix was also derived based upon nucleotide frequency (percentage conservation) at each of the 30 nucleotide positions in the alignment of promoters. For the purposes of this initial investigation only linker regions (between the -10 and -35 hexanucleotides) of $17 \mathrm{nt}$ were considered and in promoters possessing $18 \mathrm{nt}$ linkers, a non-conserved position was eliminated in the linker sequence to facilitate construction of the matrix. The secondgeneration matrix was constructed as described for the first except that all 25 verified promoter sequences (Fig. 1) were used. The program PatScan (Dsouza et al., 1997) was used to search the S. meliloti genome for matching sequences. In the case of the matrices, PatScan allows the input of a minimum threshold score such that only 30mer sequences in the genome that match or exceed the score are reported. All sequences in this work were aligned manually.

Primer extension. Primer extension reactions were conducted using total bacterial RNA and oligonucleotides listed in Table 1 as previously described (MacLellan et al., 2005). Extension and sequencing reactions were separated on $8 \%$ denaturing polyacrylamide gel containing $7 \mathrm{M}$ urea. Signals were recorded on a phosphor screen and images were processed using ImageQuant v. 5.2.

Enzyme assays and other techniques. Estimates of promoter activity were obtained by cloning DNA fragments into the transcriptional reporter vector pOT1 (Allaway et al., 2001) and measuring green fluorescent protein (GFP) fluoresence in a Tecan Safire fluorimeter. Cells were grown overnight then used to inoculate fresh volumes of $\mathrm{LB}$ broth to an $\mathrm{OD}_{600}$ of $\sim 0 \cdot 05$. Cultures were grown to an $\mathrm{OD}_{600}$ of $0 \cdot 7-0 \cdot 8$, washed once in $0 \cdot 85 \%$ saline and resuspended in 1 vol. saline. Two hundred microlitre volumes were deposited in black microtitre plates and fluorescence was measured with an excitation wavelength of $405 \mathrm{~nm}$ and an emission wavelength of $505 \mathrm{~nm}$. The optical densities of equivalent volumes were measured at $600 \mathrm{~nm}$ in clear microtitre plates. Specific fluorescence was obtained by dividing relative fluorescence by the optical density.

DNA sequencing and oligonucleotide synthesis was conducted by MOBIX lab (McMaster University, Hamilton).

\section{RESULTS}

Putative promoter sequences were predicted in two stages using a set of 11 experimentally verified promoter sequences from the existing literature in the first stage (first-generation prediction) and an expanded set of verified promoter sequences (consisting of an additional 14 promoter sequences verified in our laboratory) for the second stage (second generation) of predictions.

\section{First-generation prediction of novel promoter sequences}

Recently the characterization and mutational analysis of a promoter found upon the pSymB megaplasmid in S. meliloti led to the discovery of a small RNA gene that plays a role in 


Promoter sequences Gene Reference

\begin{tabular}{|c|c|c|c|c|c|c|}
\hline & -35 & $17-18 \mathrm{nt}$ & -10 & & & \\
\hline 1 & CTTGAC & ACTGATT-CGCGGAAGTG & GGATTC & $T$ & incA1 & (MacLellan et al. 2005) \\
\hline 2 & CTTGAC & TGTGATT-CGTGGAAATG & CGATTC & $\mathrm{T}$ & incA2 & (MacLellan et al. 2005) \\
\hline 3 & CTTGTC & TTGGGTC-AGCCTTGCCG & GTATGT & $\mathrm{T}$ & $p c k A$ & (Osteras et al. 1995) \\
\hline 4 & CTTGAC & CAAATTC-CAGTAATAAG & САATTT & $\mathrm{T}$ & $n t r A$ & (Ronson et al. 1987) \\
\hline 5 & CTTGAC & TTCGATC-GATGTTCGGG & AGAATG & A & hemA P2 & (Leong et al. 1985) \\
\hline 6 & GTTGAC & CACTGAT-CGCTTTGAAG & GAAGAA & A & hemA P1 & (Leong et al. 1985) \\
\hline 7 & CTTGAT & TCCATTAACTTCAGGGTT & CTCTAA & $\mathrm{T}$ & nodD1 & (Fisher et al. 1987) \\
\hline 8 & CTTGAT & TTCGTTAACTTCAGAGTT & СТСТAA & $\mathrm{T}$ & nod $D 2$ & -- \\
\hline 9 & CTTGCG & CGCCAGC-GCAAGCCGCG & CTAACA & $\mathrm{C}$ & $\operatorname{trp} E$ & (Bae et al. 1989) \\
\hline 10 & GTTGAC & GTGTTGGAGGGCTGGGGT & CTATAA & G & $r R N A$ & (Gufstafson et al. 2002) \\
\hline 11 & CTTGTG & AAATCAA-AAATGCTGTT & CTATAG & A & tRNA-Pro (SMc00126) & (Papp, 2004) \\
\hline 12 & ATTGAT & GGCCATAGCCGAATCGGA & GTACGT & $\mathrm{C}$ & repA2 & -- \\
\hline 13 & ATTGAC & GCTTGCG-AAGAACAGGC & GCTTAT & $\mathrm{T}$ & pcal & -- \\
\hline 14 & GTTGAC & ACGATTG-GGTCGACACG & GTATGT & G & rpmJ & this work \\
\hline 15 & CTTGAC & CGCGCCC-GAATCCCTGT & CCATGT & $\mathrm{C}$ & topA & this work \\
\hline 16 & CTTGAC & GGGATGA-AAAATTCTGG & GAATCA & $\mathrm{C}$ & $r p o D$ & this work \\
\hline 17 & CTTGAC & CCGGCCG-GTGGCCCGTT & CGATGA & G & metc & this work \\
\hline 18 & CTTGAG & АTTCСTC-АTTTCСTGAT & САATTT & $\mathrm{C}$ & ropB1 & this work \\
\hline 19 & CTTGAT & TGCGCCC-GGCCCGCCGT & GCATAA & G & $\operatorname{trps}$ & this work \\
\hline 20 & GTTGAC & GTGCGGG-CGGTTTCCCT & TTATAC & G & $\operatorname{rps} T$ & this work \\
\hline 21 & CTTGAC & ACTAGCCGGAACAGCGCC & TTATAА & G & tRNA-Met (SMc00758) & this work \\
\hline 22 & GTTGAC & AGGACGA-ATGCAGGCGG & TCATAT & A & tRNA-Tyr (SMc01378) & this work \\
\hline 23 & CTTGAC & AGATCACGGCTGTTGAGG & АААТTT & $\mathrm{C}$ & tRNA-Gly (SMc02206) & this work \\
\hline 24 & CTTGTG & GTTTTCG-CAATCGGTCT & TTATGT & A & secE & this work \\
\hline 25 & GTTGAG & TTTGCCG-ATGGCAGTCG & CTATTG & $\mathrm{C}$ & trkH & this work \\
\hline & CTTGAC & & СТАТАТ & $\mathrm{C}$ & us & \\
\hline
\end{tabular}

Fig. 1. List of experimentally verified $S$. meliloti promoter sequences. Sequences 1-11 were identified from previously published sources and were used to derive search strings and a first-generation weight-based matrix that were employed in an initial attempt to predict novel promoters in the S. meliloti genome. Sequences 12 and 13 were obtained as a result of concurrent but independent investigations in our laboratory and sequences 14-25 were promoter sequences generated by our first-generation attempt to predict novel promoters that were subsequently verified using primer extension (see Fig. 4). The consensus sequence derived from the alignment is based upon the most abundant nucleotide at each position in the -10 and -35 hexanucleotide sub-sequences. Gaps (dashes) in the sequences were arbitrarily inserted to preserve alignment at the hexanucleotides. All 25 sequences were used to generate the second-generation weight-based matrix that is described in the text.

regulating the replication of the plasmid (MacLellan et al., 2005). We noticed a striking conservation of sequence (CTTGAC) at the -35 region of the small RNA (incA1) promoter with other $S$. meliloti promoter sequences (Bae et al., 1989; Fisher et al., 1987; Leong et al., 1985; Osteras et al., 1995; Ronson et al., 1987) previously reported in the literature. We therefore compiled 11 experimentally verified promoter sequences that were related to one another (most obviously at the -35 hexanucleotide sub-sequences) with the idea of using them to predict the location of other related promoter sequences in the $S$. meliloti genome. These 11 promoter sequences are listed (and aligned) in Fig. 1 (sequences 1-11). Two strategies were employed to identify sequences in the genome that had similarity to these sequences. First, we used the program PatScan (Dsouza et al., 1997) to perform a string pattern search of the $S$. meliloti genome (see http://sequence.toulouse.inra.fr/meliloti.html) using IUPAC symbols that constituted a degenerate consensus of the verified promoter sequences and a consensus based solely upon the rRNA operon promoter sequence. Amongst over 160 hits that were within $\sim 250 \mathrm{bp}$ on the proper strand upstream of a predicted ORF we identified two hits occurring upstream of genes involved in transcription ( $s u h R, r p o D)$, twelve involved in translation [four tRNA genes (SMc01378, SMc01936, SMc00758, SMc00303), two aminoacyl synthetases (cysS, $\operatorname{trpS})$ and six ribosomal proteins (rluD, rpmE, rpmH, rpmJ, rplM, rpsT)] and hits linked with a number of other physiologically important or essential genes including $\sec E$ (protein translocation subunit), topA (topoisomerase I), two proteases ( $p \operatorname{tr} B$, SMc03769), fixN3 (cytochrome $c$ oxidase subunit), expA1 (one of a 21-gene cluster required for exopolysaccharide synthesis), and a predicted chaperone gene, grpE. Eleven hits were linked with solute transporter loci and 14 were linked 
with transcriptional regulatory protein genes. These hits are tabulated and aligned in Fig. 2.

We also constructed a weight-based matrix that we could use to assign scores to sub-sequences in the genome based on similarity to the verified promoter sequences. Best hits using this strategy were defined based on those sequences whose score equalled or exceeded an arbitrary discriminating threshold value. This first-generation weight matrix was used to scan the $S$. meliloti genome. The maximum score attainable with this matrix was 1860 and no sequence in the S. meliloti genome attained this score. Using a cutoff score of 1300 , many more candidate promoter hits were obtained, including sequences linked to genes SMc02206 (tRNA),
cspA6 (a cold-shock protein), dnaE1 (DNA polymerase subunit), pip3 (proline iminopeptidase), rpoZ (RNA polymerase omega subunit) and nuoAl (the first gene in an 11-gene cluster encoding subunits of an $\mathrm{NADH}$ dehydrogenase complex) (Fig. 2).

The hits tabulated in Fig. 2 were selected from the firstgeneration prediction stage as their function is generally well established in bacteria and several are either very important or essential to cellular viability. These fall into well-defined physiological groups such as genes involved in translation or in ribosomal structure and function. The degree of sequence conservation within the -10 and -35 sub-sequences aligned in Fig. 2 is in most cases striking and
Sequences

\begin{tabular}{llll} 
& $\mathbf{- 3 5}$ & $\mathbf{- 1 0}$ & \\
\cline { 2 - 3 } 1 & CTTGTGTTGCAAAGAC-AATGCCGTAATGC & suhR \\
2 & CTTGACGGGATGAAAA-ATTCTGGGAATAC & rpoD \\
3 & GATGGCAGCGTTCCAC-AGACGTGGCATTTT & rpoZ \\
4 & GTTGACAGGACGAATG-CAGGCGGTCATATA & tRNA (SMc01378) \\
5 & GTTGACCGGCGCAAAG-CCTGCGTGCATATG & tRNA (SMc00303) \\
6 & CTTGACACTAGCCGGAACAGCGCCTTATAAG & tRNA (SMc00758) \\
7 & GGTCGCTCCGCTGGGCCCGCATATATATAAG & tRNA (SMc01936) \\
8 & CTTGACAGATCACGGC-TGTTGAGGAAATTT & tRNA (SMc02206) \\
9 & CTTGACCGGCGCCGGG-CGACGCGCGATAAG & cysS \\
10 & CTTGATTGCGCCCGGC-CCGCCGTGCATAAG & trpS \\
11 & CTTGTCTTCCTGTTCT-TCAGGCTCGATTGC & rluD \\
12 & CTTGTTATCCCGCAGA-CCTTCATGTATAGG & rpmE \\
13 & GTTGACACGATTGGGT-CGACACGGTATGTG & rpmJ \\
14 & GTTGACGTGCGGGCGG-TTTCCCTTTATACG & rpsT \\
15 & ATTGACTTTATCGGGTTTTTGTCCCTATAAG & rpmH \\
16 & CTTGACCTTGTTTGCG-AGCTTCCGTATAAG & rpIM \\
17 & CTTGATTTGTTCCCTC-GGGAGATAGATGAT & fixN3 \\
18 & GTTGTGCACTGCGGAA-TCCACGGATATGGT & expA1 \\
19 & CTTGATCGCCGCGCTG-GTGCCCTCTATCTC & ptrB \\
20 & CTTGTCTCCGGTCCGG-TCACAGGCCAACCT & pip3 \\
21 & CTTGTCACGAAGCTCC-GAGTGTGCAATCTT & SMc03769 \\
22 & CTTGAGATTCCTCATT-TCCTGATCAATTC & ropB1 \\
23 & CTTGACCGCGCCCGAA-TCCCTGTCCATGTC & topA \\
24 & CTTGTGGTTTCGCAA-TCGGTCTTTATGTA & secE \\
25 & GTTGTTCGCCGGCCGT-TTTGATGCTATTAT & aacC \\
26 & CTTGATTTTCGGGCC-CAAACATCGATATC & grpE \\
27 & CTTGAGACCTCTTGCC-GTCCCGGGCATTGA & cspA6 \\
28 & CTTGCGCCCGGGCGCC-GGCTGCGCTAACCA & nuoA1 \\
29 & CTTGAGACTGGACAGT-TGGGACGGGAAACA & dnaE1 \\
30 & CTTGACCCGGCCGGTG-GCCCGTTCGATGAG & metC \\
31 & GTTGAGTTTGCCGATG-GCAGTCGCTATTGC & trkH \\
& &
\end{tabular}

\section{Function}

transcription

translation

cytochrome oxidase
exopolysaccharide
protease
protease
protease
outer membrane
topoisomerase
protein export
drug resistance
chaperone
cold-shock protein
NADH dehydrogenase
PollII subunit
cystathionine beta-lyase
potassium transporter

Fig. 2. Selected hits from the first-generation attempt to predict novel promoters. Sequences 3, 9, 22 and $29-31$ were predicted on the basis of a high score obtained from a weight-based matrix derived from sequences 1-11 in Fig. 1 . Sequences 7, 8 and 16-18 were predicted using a search string based upon the $r R N A$ promoter sequence (see text). All of the remaining sequences were predicted using a search string analysis of the genome with a string based upon sequences 1-11 in Fig. 1. Hits shown were selected on the basis of their potential to be important or essential genes or because they cluster with other hits into functional groupings such as genes involved in translation or ribosomal structure. Some of these hits (sequences 2, 4, 6, 8, 10, 12, 13, 14, 19, 22, 23, 24, 30 and 31) were further examined to provide additional evidence that they represent bona fide promoter sequences. 
obvious. The $5^{\prime}$ nucleotide of the -35 region is almost always a $C$ or a $G$ (as is the case with all of the input sequences used for the analyses) but the weight matrix analysis with low frequency specified some hits with an A (but never a T) in this position. One of the first indications of the broad predictive power of our analysis came with the subsequent but independent identification of two promoters in the genome (repA2 and pcaI, sequences 12 and 13 in Fig. 1) that indeed possess an A at the $5^{\prime}$ nucleotide of the -35 hexanucleotide. Thus, our sequence analysis suggested that such promoters may exist and we subsequently identified two of these promoters in the course of unrelated experimental investigations in our laboratory. Although the absolute frequency of the nucleotides found at this position may change as more promoters are identified, it seems that the hierarchy of nucleotide identity at the $5^{\prime}$ position of the -35 sub-sequence is: $C>G \gg A \gg T$. All of the 31 predicted promoters listed in Fig. 2 are of course putative and we targeted 14 of these for subsequent experimental verification.

\section{Experimental verification of predicted putative promoter sequences}

One way of providing support for a predicted promoter element is to find that the sequence is positionally conserved in the genomes of related organisms. In the case of S. meliloti, we chose A. tumefaciens as a related alphaproteobacterium since the chromosomes of these organisms show a high degree of collinearity (Wood et al., 2001). Sequences upstream of homologous genes in A. tumefaciens were compared with the $S$. meliloti intergenic sequences encompassing four predicted promoters $(r p m E$, rpoD, topA, $\sec E$ ) from the list in Fig. 2. For each locus, the intergenic sequences were manually aligned (Fig. 3) and in each case the critical -10 and -35 sub-sequences were completely conserved (except for a single nucleotide substitution in the -35 region of the $\sec E$ predicted promoters). In $\sec E$ and $r p m E$, the conserved hexanucleotide sub-sequences are flanked by rather extensive degrees of sequence conservation, but in the case of $r p o D$ and topA, the conserved -10 and -35 sub-sequences are distinct in otherwise poorly conserved regions. This suggests that these discrete regions have experienced the selective pressure that would be expected for functionally important sequences such as promoter elements. It also suggests that, with whatever facility we can predict a subset of $S$. meliloti promoters, this same ability can be extended to A. tumefaciens and probably other related genera (see Discussion).

Most often, the -10 and -35 regions of promoters are inferred from the experimental determination of the transcriptional start site for a given locus. We therefore performed primer extension reactions to determine the
rpmE
$-35$
$-10$
201
S. meliloti
TCACAAG
CGCGCGGCACTTGTTATCCCO
GCAGACCTTCATGTAT?
201
A. tumefaciens TCACAAGGGGATAGCA-GGCTCTTGTtATCTGGGCGACCTTCATGTATAGGCTCCGCATCGAATTGGGAAG

98

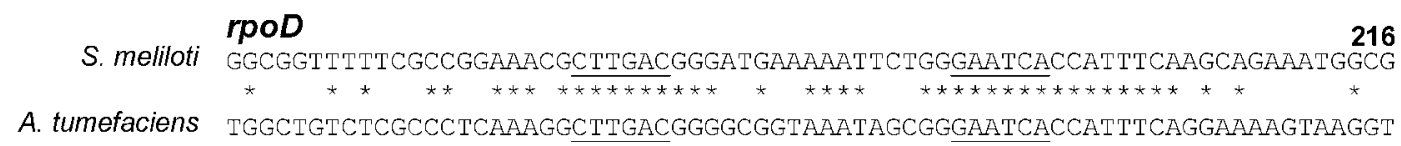

594

S. meliloti

topA

TAGCTATTTTCGATCAAACCCCTTGACCGCGCCCGAATCCCTGTCCATGTCGTGGCGTCGGATTGGCGCGC

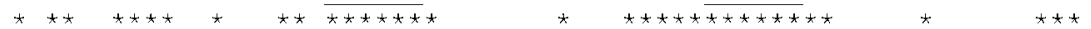

A. tumefaciens GCGATAAATTCGGGCTTGCCTCTTGACCCGCGGGCATTACCTGTCCATGTCGAATTATAAAGAAGGCCAAT

S. meliloti

$\sec E$

52

CACAAAAAAGGCTGATTGGCTCTTGTGGTTTTCGCAATCGGTCTTTATGTAGGGTCCAAACAGACACGCGG

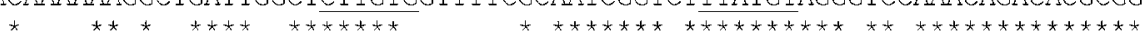

A. tumefaciens TTCGCCGAATGTCGATTTCCTCTTGTTAAAAGTGGAATCGGTGTTTATGTAGG-TCAAAACAGACACGCGG

Fig. 3. Sequence conservation between predicted $S$. meliloti promoter sequences and analogous regions from the A. tumefaciens strain C58 genome. Sequences from genetic loci (as indicated) were manually aligned, and conserved nucleotides are indicated with asterisks. Numbers indicate the distance in nucleotides between the last nucleotide shown and the predicted translational start codon for each gene. 
transcriptional start sites for 14 of the genetic loci listed in Fig. 2 and examined whether these start sites correlated with the promoter elements we predicted for these genes. The targets we examined were $r p m J, r p m E$, $p \operatorname{tr} B$, topA, rpoD, metC, ropB1, trpS, rpsT, secE, trkH, and three tRNA genes (SMc00758, SMc01378, SMc02206). The reverse transcriptase products are shown in Fig. 4(a). No extension product was visible for $\operatorname{ptr} B$ (not shown). Several of the reactions (rpmJ, topA rpoD, ropB1, SMc02206) generated multiple extension products but in every case (except for $r p o D$ ) the longest extension product (marked with an arrow) indicated a transcriptional start site that corresponds to the -10 and -35 regions that were predicted for each locus (Fig. $4 \mathrm{~b}$ ). In most cases, the shorter extension products (most of which are not shown due to space limitations) are probably due to premature termination of the reverse transcription reactions. In the case of $r p m E$, only a single extension product was observed but the transcriptional start site did not correspond to the predicted promoter element for this locus (not shown). However, we note that the extension reaction in this case terminates near a GC-rich sequence that is annotated on the $S$. meliloti genome website as a rhoindependent transcriptional terminator element. We therefore suspect that this product also represents a premature termination product. The distance between this GC-rich element and what may be the actual transcriptional start site (based on our promoter prediction) is only $17 \mathrm{nt}$ and we therefore could not design a new primer to test this hypothesis. As shown in Fig. 3, however, the sequence we predict to be the rpmE promoter is positionally conserved in the related bacterium $A$. tumefaciens, suggesting that our prediction is most likely correct. In the case of $r p o D$, an extension product (see arrow) exactly correlates with the promoter we predicted for this locus but a longer extension product was also synthesized (not shown). The nucleotide sequence upstream of this additional start site was not obviously related to the confirmed promoter but nonetheless may represent an alternative promoter at this locus. (a)
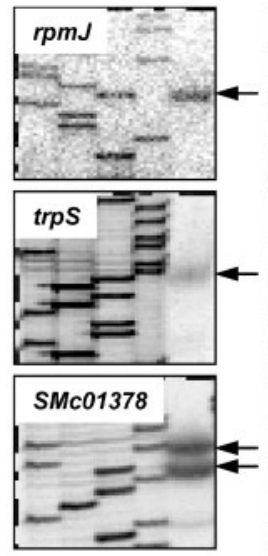

(b)

\begin{tabular}{|c|c|}
\hline rpmJ & pre \\
\hline topA & $\begin{array}{l}\text { pre } \\
\text { act }\end{array}$ \\
\hline metC & cted \\
\hline ropB1 & $\begin{array}{l}\text { cted } \\
\text { al }\end{array}$ \\
\hline $\operatorname{trps}$ & cted \\
\hline$r p o D$ & $\begin{array}{l}\text { predicted } \\
\text { actual }\end{array}$ \\
\hline$r p s T$ & $\begin{array}{l}\text { predicted } \\
\text { actual }\end{array}$ \\
\hline (2) & $\begin{array}{l}\text { predicted } \\
\text { actual }\end{array}$ \\
\hline 137 & $\begin{array}{l}\text { predicted } \\
\text { actual }\end{array}$ \\
\hline 22 & $\begin{array}{l}\text { dicted } \\
\text { ual }\end{array}$ \\
\hline $\sec E$ & . \\
\hline & te \\
\hline
\end{tabular}
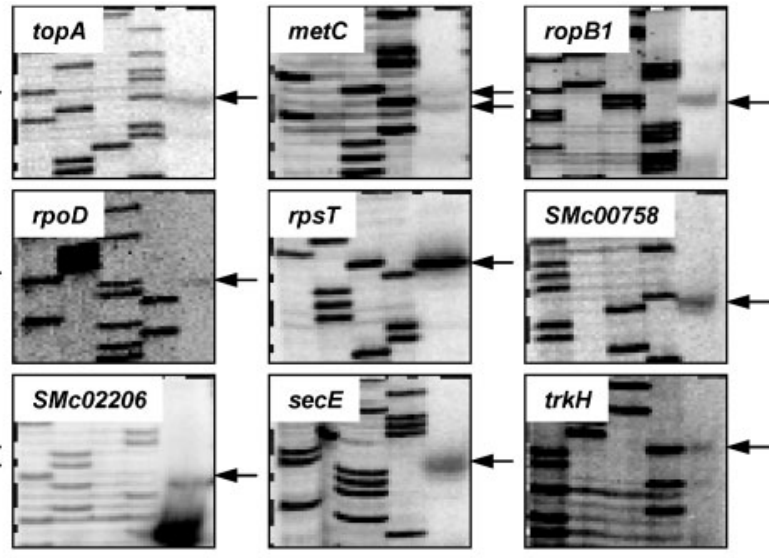

$-35$

$-10$

GTTGACACGATTGGGTCGACACGGTATGTG

GTTGACACGATTGGGTCGACACGGTATGTGCCTGCCTACTT CTTGACCGCGCCCGAATCCCTGTCCATGTC

CTTGACCGCGCCCGAATCCCTGTCCATGTCGTGGCGTCGGA CTTGACCCGGCCGGTGGCCCGTTCGATGAG $\downarrow \downarrow$ CTTGACCCGGCCGGTGGCCCGTTCGATGA GTCGGCAGCGAA CTTGAGATTCCTCATTTCCTGATCAATTTC $\downarrow$ CTTGAGATTCCTCATTTCCTGATCAATTTCGGGTCAACCGG CTTGATTGCGCCCGGCCCGCCGTGCATAAG $\downarrow$ CTTGATTGCGCCCGGCCCGCCGTGCATAAGCGCGGCGGGAT CTTGACGGGATGAAAAATTCTGGGAATCAC $\downarrow$ CTTGACGGGATGAAAAATTCTGGGAATCACCATTTCAAGCA GTTGACGTGCGGGCGGTTTCCCTTTATACG GTTGACGTGCGGGCGGTTTCCCTTTATACGCCGCCCTCAGT CTTGACACTAGCCGGAACAGCGCCTTATAAG CTTGACACTAGCCGGAACAGCGCCTTATAAGCCGCCCGACC GTTGACAGGACGAATGCAGGCGGTCATATA $\downarrow \downarrow$ GTTGACAGGACGAATGCAGGCGGTCATATACCCGGCGCAGA CTTGACAGATCACGGCTGTTGAGGAAATTTC $\downarrow$ CTTGACAGATCACGGCTGTTGAGGAAATTTCCGGTTCTGTC CTTGTGGTTTTCGCAATCGGTCTTTATGTA $\downarrow$ CTTGTGGTTTTCGCAATCGGTCTTTATGTAGGGTCCAAACA GTTGAGTTTGCCGATGGCAGTCGCTATTGC $\downarrow$ GTTGAGTTTGCCGATGGCAGTCGCTATTGCACATTGCCGCG
Fig. 4. Determination of transcriptional start sites upstream of target ORFs using primer extension. (a) Extension products using primers complementary to predicted transcripts from the gene as indicated. Arrows indicate the extension product that is consistent with the predicted promoter sequences for each locus. (b) Predicted promoter sequences (from Fig. 2) and the actual transcriptional start site (vertical arrow) for each locus determined from reactions in (a). 
This experimental approach established that 12 of 13 $(\sim 92 \%)$ of the promoter elements we predicted and targeted for verification (not including $p \operatorname{tr} B$, for which no information was obtained) are indeed genuine promoters and it is reasonable to suspect that the lone candidate that was not confirmed $(r p m E)$ may be the result of premature termination of the primer extension reaction. In a circumstance that parallels the situation in E. coli, an A residue is the most frequently (50\%) utilized transcription start nucleotide (Fig. 4b). The 12 predicted and confirmed promoter elements are listed in Fig. 1 (sequences 14-25) and all of the (now 25) verified promoter sequences were used to construct a second-generation weight matrix.

\section{Second-generation weight-based matrix}

We compiled the newly verified 12 promoter sequences (this work) with the original 11 sequences and with the two additional promoter sequences independently verified in our laboratory (sequences 12 and 13, Fig. 1) to generate a list of 25 verified promoter sequences (Fig. 1, sequences 1-25). Using these 25 verified promoter sequences we constructed a second-generation (and presumably more robust) weight matrix that we again used to scan intergenic regions in the S. meliloti genome using the program PatScan. The weight matrix in this case only identifies putative promoters that have a $17 \mathrm{nt}$ linker region between the -35 and -10 sub-sequences.

The highest possible score with this matrix is 1484 and no sequence in the genome obtained this score. Five randomly generated 30-mer sequences (with the same $\mathrm{G}+\mathrm{C}$ content as $S$. meliloti intergenic DNA: $56 \%$ ) had a mean score of only $710 \pm 59$ (SE). Of the sequences used to construct the matrix (Fig. 1) the promoter for the rRNA gene has the highest score (1376) while the promoter for the $\operatorname{trp} E$ gene has the lowest score (1116). The highest scoring sequence (with a $17 \mathrm{nt}$ linker) in the genome had a score of 1364 and was a hit linked to gene SMa0229, a hypothetical ORF whose predicted product has similarity to the translation elongation factor GreA. Hits linked to genes cysS and topA (both with a score of 1360) were the next highest scoring sequences in the genome. Using an arbitrary threshold score of $>1190,411$ hits were obtained. Ninety-five $(23 \%)$ of these hits were not obviously linked to annotated genes. Some of these hits occurred in large intergenic regions while others occurred close to annotated genes but on the opposite strand. Seven of these orphan promoter-like sequences were closely linked (within $\sim 200 \mathrm{nt}$ ) to orphan rho-independent transcriptional termination signals (that are annotated on the S. meliloti genome website), raising the possibility that together these elements may indicate the presence of small RNA genes. The number of orphan promoters closely linked with orphan terminators is likely an underestimation since a great many sequences that are good candidates for termination sequences are currently not annotated.

Eighteen and 12 hits, respectively, were closely linked with transcriptional regulatory protein genes and oxidoreductase/ dehydrogenase genes. Twenty-nine hits were linked with transporter genes. As befitting the frequency distribution of transporter types in the genome (Galibert et al., 2001), most of the hits link to $\mathrm{ABC}$ transporter genes. These, along with other selected predictions, are listed in Table 2. Most of the hits listed in Table 2 come from the second-generation weight matrix analysis but some of the hits (including several tRNA gene and ribosomal protein gene promoter predictions) are duplications from Fig. 2 (from the first round of promoter predictions) and are included solely to facilitate sequence comparison amongst genes within a functional family. Table 2 also includes 32 hits linked to genes involved in translation and RNA metabolism and other hits to physiologically important or essential genes. Except for those few that we verified using primer extension, all of the sequences in Table 2 are of course putative but we expect roughly the same prediction success rate as demonstrated from the first generation of promoter predictions (that is 12 of 13 or minimally $\sim 90 \%$ ).

\section{Characterization of the S. meliloti promoter}

Based upon the 25 verified S. meliloti promoter sequences (Fig. 1), we tabulated the frequency of each nucleotide at each of the positions delimited by the inferred -35 and -10 hexanucleotide sub-sequences and at each of 20 nucleotide positions upstream and downstream of the promoter (Fig. 5a). A plot of $\mathrm{A}+\mathrm{T}$ frequency across the regions shows a defined profile of $\mathrm{A} / \mathrm{T}$-richness at the -10 and -35 sequences. The region upstream of the -35 hexanucleotide is also frequently rich in either A or T residues and 18 of the 25 sequences carry stretches of four or more contiguous $\mathrm{A}$ or $\mathrm{T}$ residues in the $20 \mathrm{nt}$ that precede the -35 region. These sequences may function analogously to the UP elements found in the -40 to -60 region of many bacterial promoters (Aiyar et al., 1998; Ross et al., 1998). In contrast, the DNA region directly downstream of the -10 region is distinctly GC-rich, a situation reminiscent of the discriminator sequences found in some E. coli promoters (Zacharias et al., 1990, 1991). A plot of conservation (percentage abundance) of the most abundant residue at each position (Fig. 5a) shows that the -35 region is well demarcated but that the -10 sub-sequence is ill-defined and more diverse than is the -35 sequence. The -35 region has the consensus sequence CTTGAC and this assignment is supported by the facts that the $5^{\prime}$ and $3^{\prime}$ terminal $\mathrm{C}$ residues are conserved in $68 \%$ and $64 \%$ of the sequences (respectively) (Fig. 5a) while the proximal flanking nucleotides on either end of the hexanucleotide show considerably less bias. We deduce that the -10 region has the consensus sequence CTATAT but except for nucleotides 3 and $4(\mathrm{~A}, \mathrm{~T})$, which are conserved in $88 \%$ and $80 \%$ of the sequences (respectively), there is less pronounced nucleotide bias at any of the positions (see S. meliloti sequence in Fig. 5b). The overall consensus (CTTGAC_CTATAT) specifies that 19 of the 25 verified promoters listed in Fig. 1 have a linker region that consists of $17 \mathrm{nt}$. The -35 and $-10 \mathrm{~S}$. meliloti sub-sequences bear striking similarity to the canonical E. coli promoter 
Table 2. List of predicted S. meliloti promoters

\begin{tabular}{|c|c|c|c|}
\hline Sequence & Score & Gene & Function \\
\hline \multicolumn{4}{|l|}{ Transporters } \\
\hline GTTGACGGTACTTGTA-CTAATATGTATAGA & 1240 & SMa0391 & $\mathrm{ABC}$ transporter \\
\hline CGTGACAGCTACGCCA-ACCCGTGGCATAGG & 1200 & SMa0451 & $\mathrm{ABC}$ transporter \\
\hline GTTGACGCATGTCAGG-AAACATTTTAGATC & 1200 & SMa0079 & ABC transporter \\
\hline GTTGACACTGTGAACT-TTTGATGCAACAAA & 1196 & SMa0953 & $\mathrm{ABC}$ transporter \\
\hline CTTGACGCTCGTTAGC-TTTGGTACTACCAG & 1216 & SMa1337 & $\mathrm{ABC}$ transporter \\
\hline CTTGACTGCTAATAAT-ATTAGCGCAAGGTT & 1220 & $\mathrm{SMb} 21345$ & $\mathrm{ABC}$ transporter \\
\hline GTTGACAGCTTGCGGA-AACTCTGACATTTA & 1208 & SMb20902 & $\mathrm{ABC}$ transporter \\
\hline CTTGACGGCGCCCGGA-TAAAATGGAATACA & 1316 & $\operatorname{moc} B$ & $\mathrm{ABC}$ transporter \\
\hline GTTGACAAACGGACGG-TCTGAATCGATCAT & 1204 & SMb20155 & $\mathrm{ABC}$ transporter \\
\hline ATTGATTTGTGCTCTC-CGACGCGGAATAGT & 1200 & SMb20263 & $\mathrm{ABC}$ transporter \\
\hline GTTGACATTTGTCGAG-CCATGTTCGAATTA & 1208 & SMb20315 & $\mathrm{ABC}$ transporter \\
\hline ATTGACATGATTTGGG-AACCGGATCATCGT & 1200 & SMb20427 & $\mathrm{ABC}$ transporter \\
\hline CTTGACACATCTGGAC-TATTGAACAAGAAA & 1204 & SMb21221 & $\mathrm{ABC}$ transporter \\
\hline ATTGACATTTCAGCTC-AATCGGTGAGTAAT & 1204 & $s m o E$ & $\mathrm{ABC}$ transporter \\
\hline CTTGGCAGCAGTGCCG-TCCTACTGCATGTT & 1228 & Y00717 & $\mathrm{ABC}$ transporter \\
\hline CTTGACACCGCGTCAG-TAGGATTTACTAAT & 1252 & Y03131 & $\mathrm{ABC}$ transporter \\
\hline СTTGACTCTTTCTCTG-CAGCGATGGAGTGT & 1212 & $\operatorname{agle}$ & $\mathrm{ABC}$ transporter \\
\hline ATTGCCCGGACCTCAG-ATCCGGGCAATTTT & 1224 & $\operatorname{pot} G$ & $\mathrm{ABC}$ transporter \\
\hline СTTGACCTTACCATTG-TTGGAAGGACTATC & 1232 & actP & P-type $\mathrm{Cu}^{2+}$ transporter \\
\hline CTTGACCTTCCCATTA-TTGGAAGGATTATG & 1204 & act $U 2$ & P-type $\mathrm{Cu}^{2+}$ transporter \\
\hline CTTGAATCACACGGGC-TTTGCGCGTATAAC & 1236 & SMa1664 & Multi-drug efflux resistance \\
\hline TTTGACTTTGGTCATC-CGCTGGTCGAATTC & 1200 & SMa1691 & trkH-like cation transporter \\
\hline CTTGATTCAAAGCAAG-TTGAGTGGAAGGAT & 1196 & $k e f B 2$ & $\mathrm{~K}^{+}$efflux \\
\hline CTTGACCCGGGATTCA-GCGTCCCCTATTTC & 1200 & SMb20361 & Voltage-gated transporter \\
\hline CTTGAGGCAGGCCCGC-CCTTCTTCTATGTC & 1272 & phaA2 & Cation efflux \\
\hline CTTGGCAGCCGTCCGG-AAAATAGTCATAAA & 1200 & phaAl & Cation efflux \\
\hline CTTGACGGGAATCGAA-TACTGGAGAACTCT & 1212 & $a q p Z 1$ & Aquaporin \\
\hline ATTGATTTTGCGCCGG-GGTTGCGTCATATC & 1236 & corA2 & $\mathrm{Mg}^{2+}, \mathrm{Co}^{2+}$ transporter \\
\hline CTTGACGGAACCGCGC-GCCGGCGCTCGAAT & 1220 & SMc04404 & Amino acid efflux \\
\hline \multicolumn{4}{|l|}{ RNA metabolism } \\
\hline CTGGAAGAGAATCATG-CCCGGTGGCATGTC & 1192 & SMa0022 & Sec-tRNA (TGA) \\
\hline CTTGACACTAGCCGGAACAGCGCCTTATAAG & 1344 & SMc00758 & Met-tRNA (CAT) \\
\hline GGTCGCTCCGCTGGGCCCGCATATATATAAG & 984 & SMc01936 & Asp-tRNA (GTC) \\
\hline GTTGACAGGACGAATG-CAGGCGGTCATATA & 1284 & SMc01378 & Tyr-tRNA (GTA) \\
\hline CTTGAAAGCGTCCGGC-AAACTGAATATTAA & 1224 & SMc01381 & Trp-tRNA (CCA) \\
\hline CTTGACTGAATCGCCT-ATGTGCCTGATTTA & 1224 & SMc00957 & Leu-tRNA (TAA) \\
\hline GTTGACCGGCGCAAAG-CCTGCGTGCATATG & 1276 & SMc00303 & Lys-tRNA (TTT) \\
\hline CTTGCGGGAATCCGAA-GCCCTCTCTATAAG & 1224 & SMc00304 & Glu-tRNA (TTC) \\
\hline CTTGCATGAACCCGAT-GCCCTCTCTATAAG & 1212 & SMc00305 & Glu-tRNA (CTC) \\
\hline CTTGCGAGAATCCGAA-GCGCTCTCTATAAG & 1228 & SMc00306 & Glu-tRNA (TTC) \\
\hline CTTGCCAGCGGCAGAA-ACTGCCGCTATGAA & 1264 & SMc03779 & Ser-tRNA (CGA) \\
\hline CTTGAAATCTCGCCGC-TTTGCGTTTATCAG & 1264 & SMc02183 & Thr-tRNA (CGT) \\
\hline CTTGACAGATCACGGC-TGTTGAGGAAATTT & 1208 & SMc02206 & Gly-tRNA (CCC) \\
\hline CTTGACCGGCGCCGGG-CGACGCGCGATAAG & 1360 & cysS & tRNA synthetase \\
\hline CTTGACAAAACCGGCG-CATCATGCGCTTTT & 1212 & $\operatorname{aspS}$ & tRNA synthetase \\
\hline GTTGACTTGCCCCGGG-CCTTCCGGTTTACA & 1200 & vals & tRNA synthetase \\
\hline CTTGACCCTCGCCCTG-CGGCATGGCACCTG & 1208 & $g l y Q$ & tRNA synthetase \\
\hline CTTGATTGCGCCCGGC-CCGCCGTGCATAAG & 1316 & $\operatorname{trpS}(\mathrm{P} 1)$ & tRNA synthetase \\
\hline CTTGTGGAGGGCCCGG-CTCCAGTTGATATC & 1220 & $\operatorname{trpS}(\mathrm{P} 2)$ & tRNA synthetase \\
\hline CTTGCCGTGACGGGCG-CAAGTTTCTATATG & 1272 & $\operatorname{trm} U$ & tRNA methyltransferase \\
\hline CTTGTCTTCCTGTTCT-TCAGGCTCGATTGC & 1168 & rluD & Ribosomal protein \\
\hline CTTGTTATCCCGCAGA-CCTTCATGTATAGG & 1172 & $r p m E$ & Ribosomal protein \\
\hline
\end{tabular}


Table 2. cont.

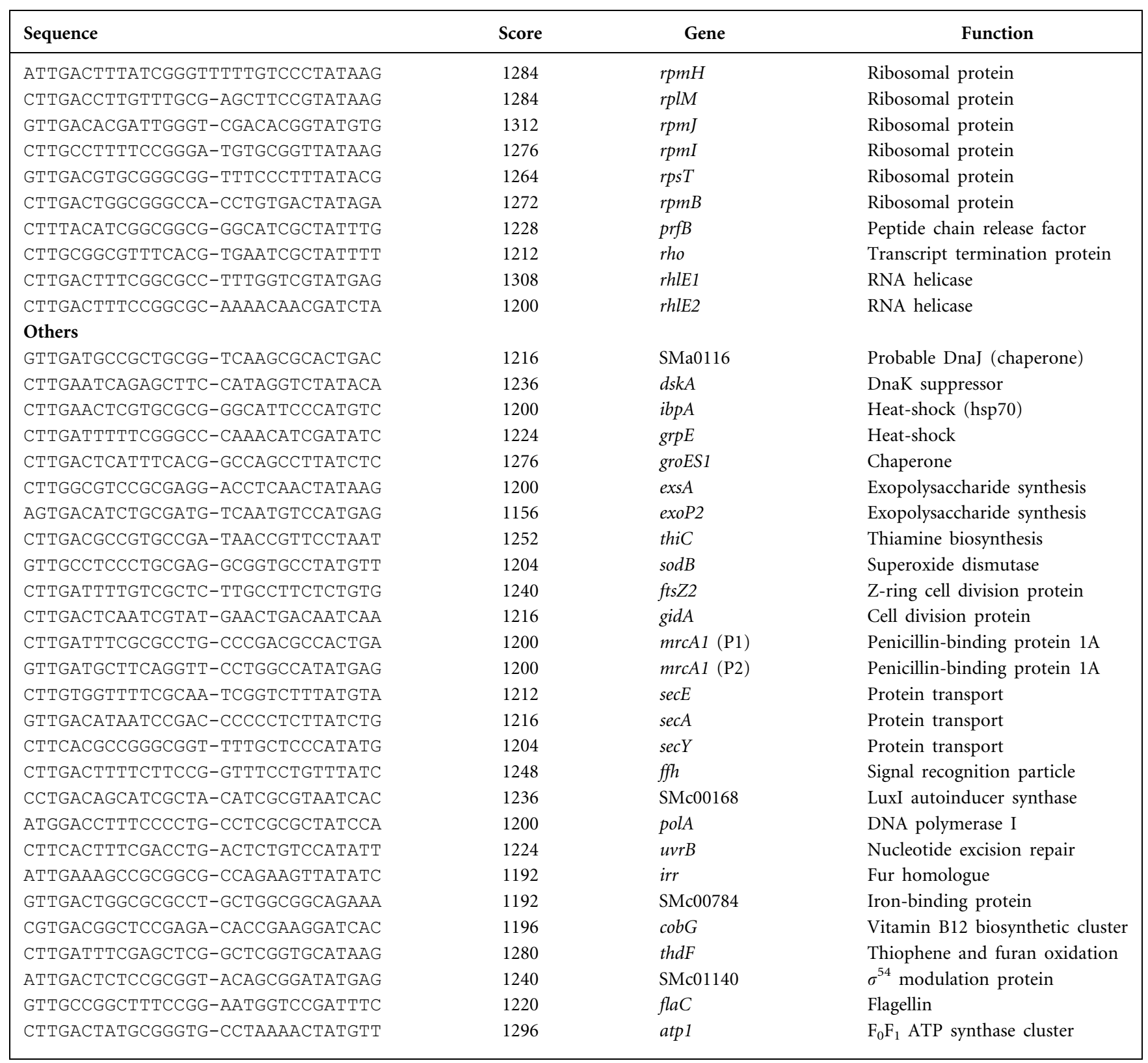

consensus (Harley \& Reynolds, 1987; Lisser \& Margalit, 1993) and these sequences are directly compared in Fig. 5(b). At the -35 sub-sequence, both E. coli and $S$. meliloti promoters share a core sequence of TTGAC but they are shifted or skewed relative to one another by a $1 \mathrm{nt}$ step, at least as evidenced by the conservation of nucleotides in these regions (Fig. 5b).

Given the significant similarity between these $S$. meliloti promoters and the canonical E. coli promoter, we cloned approximately $200 \mathrm{bp}$ regions from upstream of the genes $r p o D, s e c E, r p m J, r p m E$, ropB1, SMc1378, rpsT and topA (see list in Fig. 2) into the $g f p$ transcriptional reporter pOT1 and promoter activity was measured in E. coli, A. tumefaciens and S. meliloti. As a control for these experiments, we used the incA promoter, which is known to be expressed in E. coli (MacLellan et al., 2005). Most of the promoters were highly active in both $A$. tumefaciens and $S$. meliloti, but other than the control incA, none were appreciably active in $E$. coli. In this host, the most active promoter belonged to $r p m E$ (with activity $1 \cdot 9$-fold above background) (Table 3 ). We expect that several of these housekeeping genes encode factorindependent promoters and thus should technically be capable of activity in heterologous hosts. In some instances, however, promoter activity may be dependent on hostspecific transcriptional regulators not present in E. coli. Nevertheless, this survey is quite consistent with the common finding in our laboratory that $S$. meliloti promoters 
(a)

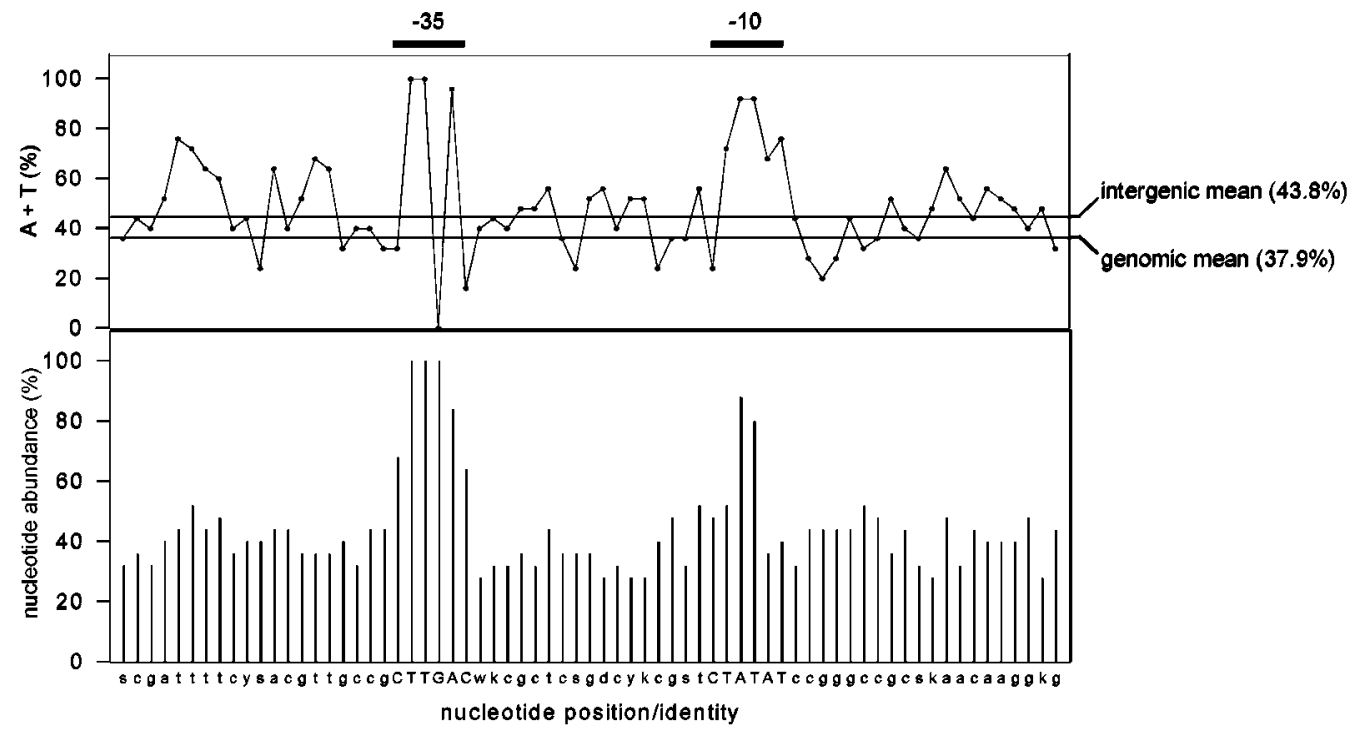

(b)

\begin{tabular}{|c|c|c|c|c|c|c|c|c|c|c|c|c|c|c|c|}
\hline E. coli & $\begin{array}{l}29 \\
9\end{array}$ & $\begin{array}{l}69 \\
T\end{array}$ & $\begin{array}{c}79 \\
T\end{array}$ & $\begin{array}{r}61 \\
\text { G }\end{array}$ & $\begin{array}{r}56 \\
\mathrm{~A}\end{array}$ & ${ }^{54} \mathrm{C}$ & $\begin{array}{r}54 \\
\mathrm{~A}\end{array}$ & -- & $\begin{array}{r}35 \\
9\end{array}$ & $\begin{array}{l}77 \\
\text { T }\end{array}$ & $\begin{array}{l}76 \\
\text { A }\end{array}$ & $\begin{array}{l}60 \\
\mathbf{T}\end{array}$ & $\begin{array}{l}61 \\
\mathrm{~A}\end{array}$ & $\begin{array}{l}56 \\
\mathrm{~A}\end{array}$ & $\begin{array}{l}82 \\
\text { T }\end{array}$ \\
\hline S. meliloti & $\mathrm{C}$ & $\mathbf{T}$ & $\mathbf{T}$ & G & A & C & $\mathrm{W}$ & -- & $\mathrm{C}$ & $\mathbf{T}$ & $\mathbf{A}$ & $\mathbf{T}$ & $\mathbf{A}$ & $\mathbf{T}$ & C \\
\hline & 68 & 100 & 100 & 100 & 84 & 64 & 28 & & 48 & 52 & 88 & 80 & 36 & 40 & 32 \\
\hline
\end{tabular}

Fig. 5. Analysis of the S. meliloti promoter. (a) Frequency of most abundant nucleotide at each position in 25 experimentally verified promoters (see Fig. 1). The sequence plotted ranges from $20 \mathrm{nt}$ upstream of the -35 sub-sequence to $20 \mathrm{nt}$ downstream of the -10 sub-sequence. The most abundant nucleotide(s) at each position is labelled ( -35 and -10 nucleotides are capitalized). $A+T$ abundance at each position is shown. Reference lines indicate the mean genomic and intergenic region $A+T$ content. (b) Nucleotide frequency at each $E$. coli and $S$. meliloti -35 and -10 position as deduced from sequences 1-25 in Fig. 1. Consensus nucleotides are in bold capitals; flanking nucleotides are in lower case.

rarely display activity in E. coli, a circumstance that provided a major motivation towards better defining at least a subset of $S$. meliloti promoter sequences.

Despite the conservation of core sequence (TTGAC) between the E. coli and S. meliloti -35 hexanucleotides we have concluded that the $S$. meliloti consensus is completed by a $5^{\prime}$ C residue (CTTGAC) as opposed to the E. coli consensus, which is completed by a $3^{\prime}$ A residue (TTGACA) based on the frequency of conserved nucleotides. Using site-directed mutagenesis on two $S$. meliloti promoters (the incA1 and incA2 gene promoters) we sought to provide experimental support for the idea that the $5^{\prime} \mathrm{C}$ residue of the consensus is functionally important for promoter activity and, conversely, that the $3^{\prime}$ nucleotide most proximal to CTTGAC has little functional importance. To do this, we cloned both promoter regions into the $g f p$ transcriptional reporter vector POT1 and monitored promoter activity as specific fluorescence in both A. tumefaciens and E. coli cells. We found it necessary to use the closely related bacterium A. tumefaciens as host for the test plasmids because the products expressed from the incA1 and incA2 promoters ( $\sim 56 \mathrm{nt}$ untranslated RNAs) mediate incompatibility against the resident megaplasmids of $S$. meliloti (MacLellan et al., 2005). We know from this and previous work that patterns of expression in A. tumefaciens and $S$. meliloti are comparable.

As shown in Table 4, the incA1 (pTH1560) and incA2 (pTH1982) promoters are highly active in both A. tumefaciens and E. coli. Mutations in the nucleotide $3^{\prime}$ of the consensus (pTH1985 and pTH1983, respectively) have little or no impact on promoter activity in both species. We expected this result for A. tumefaciens cells since that nucleotide falls outside the consensus hexanucleotide and is less biased than the other adjacent nucleotides. In the case of E. coli, however, we found this surprising since we expected that a $\mathrm{T}$ in that nucleotide position (which is found much less frequently than an $\mathrm{A}$ in that position) might lower activity if E. coli RNA polymerase continued to recognize 
Table 3. Activity of selected S. meliloti promoters in parental or heterologous host strains

\begin{tabular}{|lccc|}
\hline \multirow{2}{*}{ Promoter $^{*}$} & \multicolumn{2}{c|}{ Mean promoter activity \pm SD (fold above background) $\dagger$} \\
\cline { 2 - 4 } & \multicolumn{1}{c}{ E. coli } & A. tumefaciens & S. meliloti \\
\hline pOT1 & $307 \pm 14(1)$ & $168 \pm 5(1)$ & $115 \pm 7(1)$ \\
rpoD & $478 \pm 4(1 \cdot 6)$ & $1481 \pm 49(8 \cdot 8)$ & $4405 \pm 9(38 \cdot 3)$ \\
secE & $520 \pm 20(1 \cdot 7)$ & $8971 \pm 95(53 \cdot 4)$ & $25003 \pm 253(217 \cdot 4)$ \\
$r p m J$ & $237 \pm 16(\leqslant 0)$ & $14973 \pm 1302(89 \cdot 1)$ & $32452 \pm 1091(282 \cdot 2)$ \\
rpmE & $592 \pm 4(1 \cdot 9)$ & $4101 \pm 131(24 \cdot 4)$ & $14833 \pm 276(129 \cdot 0)$ \\
ropB1 & $373 \pm 9(1 \cdot 2)$ & $984 \pm 66(5 \cdot 9)$ & $1803 \pm 44(15 \cdot 7)$ \\
SMc1378 & $405 \pm 12(1 \cdot 3)$ & $19358 \pm 685(115 \cdot 2)$ & $82752 \pm 2929(719 \cdot 6)$ \\
$r p s T$ & $426 \pm 17(1 \cdot 4)$ & $9665 \pm 84(57 \cdot 5)$ & $48076 \pm 1643(418 \cdot 1)$ \\
topA & $433 \pm 24(1 \cdot 4)$ & $1567 \pm 55(9 \cdot 3)$ & $2854 \pm 63(24 \cdot 8)$ \\
incA $\ddagger$ & $34335 \pm 405(111 \cdot 8)$ & $1986 \pm 73(11 \cdot 8)$ & $\mathrm{NAS}$ \\
\hline
\end{tabular}

${ }^{\star}$ The test plasmid was pOT1 or pOT1 carrying the indicated gene promoter (see text).

†GFP activity values reflect data from three replicates in the indicated hosts. Background activity is from cells carrying the reporter vector, pOT1.

$¥$ The DNA fragment containing the small RNA gene incA promoter also carries the transcriptional terminator for the gene. This terminator is poorly recognized in E. coli but efficiently terminates in $A$. tumefaciens. Thus, the activity for the incA promoter in A. tumefaciens will be about $10-20 \%$ of the actual promoter activity.

§NA, Not applicable. Since expression of the full-length incA gene mediates incompatibility in S. meliloti, the incA promoter was not tested in this background.

the E. coli consensus hexanucleotide that is incidentally formed in plasmids pTH1560 and pTH1983 but not plasmids pTH1985 and pTH1982. In all cases, when the inferred 5 ' nucleotide of the $S$. meliloti consensus (CTTGAC) was mutated to form TTTGAC (plasmids pTH1991 and pTH1984), promoter activity was significantly affected. For reasons we have not determined, the influence of the mutation is much more dramatic in the incA2 promoter than in the incA1 promoter. Although the incA1 and incA2 promoter regions are highly similar, sequence differences elsewhere in the promoters are presumably responsible for this effect. In any case, the weight matrix analysis suggests that the -35 hexanucleotide rarely if ever begins with a $\mathrm{T}$ residue and this is consistent with the dramatic loss of promoter activity from promoters that carry this nucleotide in that position.

Table 4. Mutational analysis of $S$. meliloti promoter -35 sub-sequence

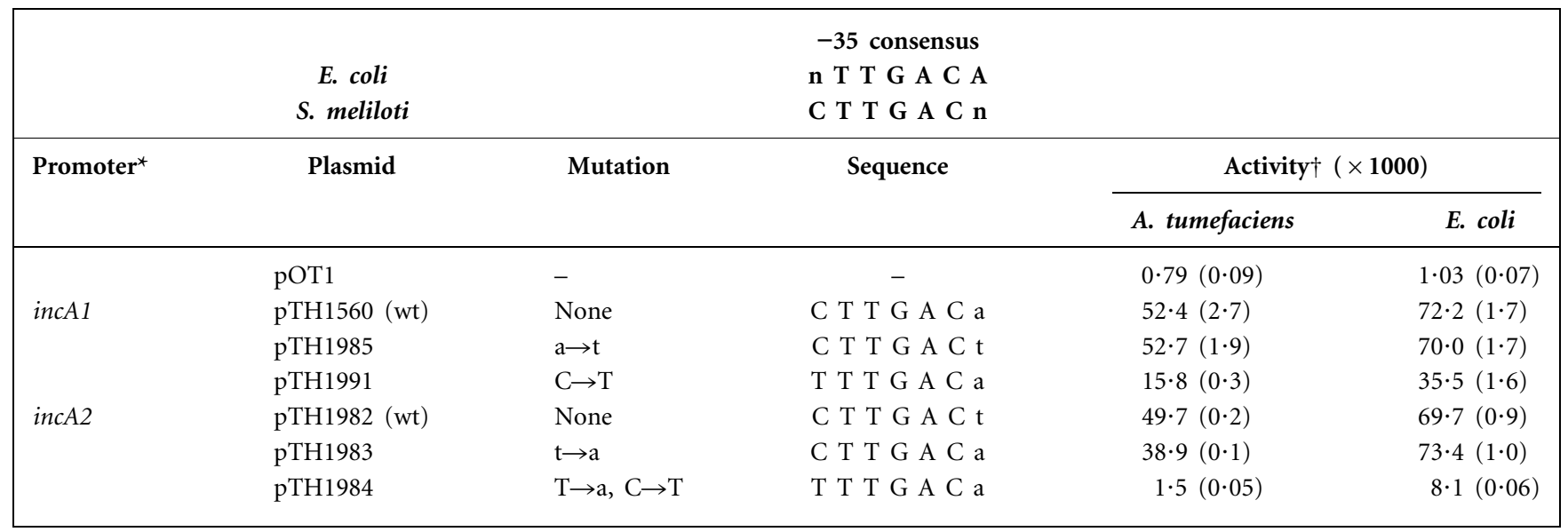

${ }^{\star}$ Gene promoter region (as depicted in Fig. 1) cloned into transcriptional reporter vector pOT1 (as described in Table 1).

$\dagger$ Activity measured as specific GFP fluorescence and reported as the mean of triplicate readings ( \pm SE). A. tumefaciens was used as a surrogate host for the plasmids as some retain the ability to mediate incompatibility against the resident megaplasmids in $S$. meliloti (see Methods). 


\section{DISCUSSION}

The ability to predict promoter elements in the alphaproteobacteria is only now emerging as the result of the accumulation of experimentally verified promoter sequences and access to the genomic sequences of these bacteria. Except for the $\sigma^{54}$ promoters (Dombrecht et al., 2002), we are aware of no concerted effort to predict promoters in any members of the group. Our effort represents a fundamental first step in this direction and as a result of this work we report the experimentally verified sequences of an additional $12 \mathrm{~S}$. meliloti promoters. As more sequences are verified, this work can be extended and refined. We provide a list (Table 2 ) of nearly 100 predicted promoters but these represent only a fraction of sequences in the $S$. meliloti genome that appear to be related to the consensus we have defined in this work.

The structure demonstrated for $S$. meliloti promoters appears to be conserved amongst other bacteria in the alphaproteobacteria for the following reasons: (1) we showed that the promoter sequences for the rpmE, rpoD, topA and secE genes were conserved between $S$. meliloti and A. tumefaciens (Fig. 3); (2) homologues of the incA1 and $i n c A 2$ genes whose promoters have been verified have homologues in A. tumefaciens, Rhizobium etli, R. leguminosarum and Brucella spp. (Chai \& Winans, 2005; Dombrecht et al., 2002; Izquierdo et al., 2005; MacLellan et al., 2005; Venkova-Canova et al., 2004) and their promoters are nearly identical; and (3) a small compilation (eight genes) of Bradyrhizobium japonicum promoters (Beck et al., 1997) demonstrated the same general pattern of nucleotide conservation as described in this work.

We have not experimentally deduced which sigma factor recognizes the confirmed and putative promoters that have been predicted in this work. However, the sigma factor is likely to be $r p o D$, the vegetative sigma factor, since many of the confirmed and predicted loci associated with these promoters encode products required for translation and transcription, and other essential or important functions.

In this work we have identified consensus -10 and -35 hexameric promoter sequences defining what we believe to be a subset of $S$. meliloti promoters. Further experimental work will be required to better define these sequences from a functional point of view and to determine whether the E. coli paradigm of conserved hexamers is appropriate for S. meliloti promoters, particularly with regard to the poorly conserved -10 region. From the perspective of predicting related sequences (putative promoters) in the alphaproteobacteria, however, the identification of patterns of nucleotide conservation described in this work is a productive first step towards more effective promoter prediction in these bacteria.

\section{ACKNOWLEDGEMENTS}

This work was supported with funding from Genome Canada through the Ontario Genomics Institute and with funding from the Ontario research and development challenge fund. The work was initially supported by a grant from the Natural Sciences and Engineering Research Council to T.M.F. We are grateful to Marie Elliot, Alison Cowie and Richard Morton for input and comments on the manuscript.

\section{REFERENCES}

Aiyar, S. E., Gourse, R. L. \& Ross, W. (1998). Upstream A-tracts increase bacterial promoter activity through interactions with the RNA polymerase alpha subunit. Proc Natl Acad Sci U S A 95, 14652-14657.

Allaway, D., Schofield, N. A., Leonard, M. E., Gilardoni, L., Finan, T. M. \& Poole, P. S. (2001). Use of differential fluorescence induction and optical trapping to isolate environmentally induced genes. Environ Microbiol 3, 397-406.

Argaman, L., Hershberg, R., Vogel, J., Bejerano, G., Wagner, E. G., Margalit, H. \& Altuvia, S. (2001). Novel small RNA-encoding genes in the intergenic regions of Escherichia coli. Curr Biol 11, 941-950.

Bae, Y. M., Holmgren, E. \& Crawford, I. P. (1989). Rhizobium meliloti anthranilate synthase gene: cloning, sequence, and expression in Escherichia coli. J Bacteriol 171, 3471-3478.

Beck, C., Marty, R., Klausli, S., Hennecke, H. \& Gottfert, M. (1997). Dissection of the transcription machinery for housekeeping genes of Bradyrhizobium japonicum. J Bacteriol 179, 364-369.

Chai, Y. \& Winans, S. C. (2005). A small antisense RNA downregulates expression of an essential replicase protein of an Agrobacterium tumefaciens Ti plasmid. Mol Microbiol 56, 1574-1585.

Chen, S., Lesnik, E. A., Hall, T. A., Sampath, R., Griffey, R. H., Ecker, D. J. \& Blyn, L. B. (2002). A bioinformatics based approach to discover small RNA genes in the Escherichia coli genome. Biosystems 65, 157-177.

DelVecchio, V. G., Kapatral, V., Elzer, P., Patra, G. \& Mujer, C. V. (2002). The genome of Brucella melitensis. Vet Microbiol 90, 587-592.

Dombrecht, B., Marchal, K., Vanderleyden, J. \& Michiels, J. (2002). Prediction and overview of the RpoN-regulon in closely related species of the Rhizobiales. Genome Biol 3, RESEARCH0076.

Dsouza, M., Larsen, N. \& Overbeek, R. (1997). Searching for patterns in genomic data. Trends Genet 13, 497-498.

Fisher, R. F., Brierley, H. L., Mulligan, J. T. \& Long, S. R. (1987). Transcription of Rhizobium meliloti nodulation genes. Identification of a nodD transcription initiation site in vitro and in vivo. J Biol Chem 262, 6849-6855.

Galibert, F., Finan, T. M., Long, S. R. \& 53 other authors (2001). The composite genome of the legume symbiont Sinorhizobium meliloti. Science 293, 668-672.

Gustafson, A. M., O'Connell, K. P. \& Thomashow, M. F. (2002). Regulation of Sinorhizobium meliloti $1021 \mathrm{rrnA}$-reporter gene fusions in response to cold shock. Can J Microbiol 48, 821-830.

Halling, S. M., Peterson-Burch, B. D., Bricker, B. J., Zuerner, R. L., Qing, Z., Li, L. L., Kapur, V., Alt, D. P. \& Olsen, S. C. (2005). Completion of the genome sequence of Brucella abortus and comparison to the highly similar genomes of Brucella melitensis and Brucella suis. J Bacteriol 187, 2715-2726.

Harley, C. B. \& Reynolds, R. P. (1987). Analysis of E. coli promoter sequences. Nucleic Acids Res 15, 2343-2361.

Izquierdo, J., Venkova-Canova, T., Ramirez-Romero, M. A., TellezSosa, J., Hernandez-Lucas, I., Sanjuan, J. \& Cevallos, M. A. (2005). An antisense RNA plays a central role in the replication control of a repC plasmid. Plasmid 54, 259-277. 
Kaneko, T., Nakamura, Y., Sato, S. \& 14 other authors (2002). Complete genomic sequence of nitrogen-fixing symbiotic bacterium Bradyrhizobium japonicum USDA110 (supplement). DNA Res 9, 225-256.

Leong, S. A., Williams, P. H. \& Ditta, G. S. (1985). Analysis of the $5^{\prime}$ regulatory region of the gene for delta-aminolevulinic acid synthetase of Rhizobium meliloti. Nucleic Acids Res 13, 5965-5976.

Lisser, S. \& Margalit, H. (1993). Compilation of E. coli mRNA promoter sequences. Nucleic Acids Res 21, 1507-1516.

MacLellan, S. R., Smallbone, L. A., Sibley, C. D. \& Finan, T. M. (2005). The expression of a novel antisense gene mediates incompatibility within the large repABC family of alpha-proteobacterial plasmids. Mol Microbiol 55, 611-623.

Osteras, M., Driscoll, B. T. \& Finan, T. M. (1995). Molecular and expression analysis of the Rhizobium meliloti phosphoenolpyruvate carboxykinase ( $p c k A)$ gene. J Bacteriol 177, 1452-1460.

Papp, P. P. (2004). tRNA gene containing an attachment site of a temperate phage is functional: proof by converting attB to amber suppressor. GenBank accession no. AJ698943.

Paulsen, I. T., Seshadri, R., Nelson, K. E. \& 28 other authors (2002). The Brucella suis genome reveals fundamental similarities between animal and plant pathogens and symbionts. Proc Natl Acad Sci U S A 99, 13148-13153.
Ronson, C. W., Nixon, B. T., Albright, L. M. \& Ausubel, F. M. (1987). Rhizobium meliloti ntrA (rpoN) gene is required for diverse metabolic functions. J Bacteriol 169, 2424-2431.

Ross, W., Aiyar, S. E., Salomon, J. \& Gourse, R. L. (1998). Escherichia coli promoters with UP elements of different strengths: modular structure of bacterial promoters. J Bacteriol 180, 5375-5383.

Thony, B. \& Hennecke, H. (1989). The $-24 /-12$ promoter comes of age. FEMS Microbiol Rev 5, 341-357.

Venkova-Canova, T., Soberon, N. E., Ramirez-Romero, M. A. \& Cevallos, M. A. (2004). Two discrete elements are required for the replication of a repABC plasmid: an antisense RNA and a stem-loop structure. Mol Microbiol 54, 1431-1444.

Wood, D. W., Setubal, J. C., Kaul, R. \& 49 other authors (2001). The genome of the natural genetic engineer Agrobacterium tumefaciens C58. Science 294, 2317-2323.

Zacharias, M., Goringer, H. U. \& Wagner, R. (1990). The signal for growth rate control and stringent sensitivity in E. coli is not restricted to a particular sequence motif within the promoter region. Nucleic Acids Res 18, 6271-6275.

Zacharias, M., Theissen, G., Bradaczek, C. \& Wagner, R. (1991). Analysis of sequence elements important for the synthesis and control of ribosomal RNA in E coli. Biochimie 73, 699-712. 